\title{
PREVALÊNCIA E PADRÃO DE DISTRIBUIÇÃO DAS DOENÇAS CEREBROVASCULARES EM 242 IDOSOS, PROCEDENTES DE UM HOSPITAL GERAL, NECROPSIADOS EM BELO HORIZONTE, MINAS GERAIS, NO PERÍODO DE 1976 A 1997
}

\author{
José Eymard H. Pittella ${ }^{1}$, Juliana Elias Duarte ${ }^{2}$
}

\begin{abstract}
RESUMO - Objetivo: Descrever a prevalência e os tipos das doenças cerebrovasculares (DCVs) em indivíduos idosos necropsiados. Método: Foram consultados os laudos neuropatológicos de 242 pacientes com idade igual ou superior a 61 anos, procedentes em sua maioria do Hospital das Clínicas da Universidade Federal de Minas Gerais, em Belo Horizonte, Minas Gerais, e necropsiados consecutivamente no período 1976 a 1997. Resultados: Os principais grupos de doenças do sistema nervoso central (SNC) foram representados por DCVs (71,9\%), infecções $(12,4 \%)$, neoplasias $(7,1 \%)$, traumatismos crânio-encefálicos $(3,7 \%)$, doenças nutricionais $(2,5 \%)$ e doenças degenerativas $(1,7 \%)$. As DCVs mais frequentes foram: aterosclerose $(61,2 \%)$, doença cerebrovascular hipertensiva $(25,6 \%)$ e infarto cerebral $(14,9 \%)$. Observou-se aumento da frequência e da gravidade da aterosclerose e da frequência da doença cerebrovascular hipertensiva com o avançar da idade. Houve associação significativa entre doença cerebrovascular hipertensiva e aterosclerose. As DCVs foram clinicamente sintomáticas e as responsáveis diretas pelo óbito em 42,7\% e 17,3\% dos pacientes, respectivamente. Conclusão: As DCVs constituíram o principal grupo de doenças do SNC no idoso. A aterosclerose e a doença cerebrovascular hipertensiva foram as principais doenças deste grupo, notando-se aumento de sua frequência com o avançar da idade e associação significativa entre ambas.
\end{abstract}

PALAVRAS-CHAVE: doença cerebrovascular, idoso, necropsia.

Prevalence and types of cerebrovascular diseases in 242 hospitalized elderly patients, autopsied in Belo Horizonte, Minas Gerais, Brazil, from 1976 to 1997

\begin{abstract}
Objective: To describe the prevalence and the types of cerebrovascular diseases (CVDs) in autopsied elderly individuals. Method: Consecutive clinical charts and neuropathological reports of 242 patients aged 61 years or older were reviewed. The patients died in Hospital das Clínicas, Federal University of Minas Gerais, in Belo Horizonte, Minas Gerais, Brazil, from 1976 to 1997. Results: The prevalent diseases of the central nervous system (CNS) found in decreasing order were: CVDs (71.9\%), infections (12.4\%), neoplasms (7.1\%), head trauma (3.7\%), nutritional diseases (2.5\%) and degenerative diseases (1.7\%). The most common CVDs were cerebral atherosclerosis (61.2\%), hypertensive cerebrovascular disease (25.6\%) and cerebral infarct (14.9\%). There was an increase in prevalence and severity of atherosclerosis and an increase in prevalence of hypertensive cerebrovascular disease with advancing age. A significant association between hypertensive cerebrovascular disease and atherosclerosis was found. The CVDs patients had clinical stroke and this was the direct cause of death in $42,7 \%$ and $17,3 \%$ of the cases, respectively. Conclusion: The CVDs were the most prevalent group of diseases of the CNS in elderly patients. Atherosclerosis and hypertensive cerebrovascular disease were the most common CVDs, and its prevalence increased with advancing age. Hypertensive cerebrovascular disease was significantly associated with atherosclerosis.
\end{abstract}

KEY WORDS: cerebrovascular disease, elderly, autopsy.

\footnotetext{
Laboratório de Neuropatologia, Departamento de Anatomia Patológica e Medicina Legal, Faculdade de Medicina, Universidade Federal de Minas Gerais, Belo Horizonte MG, Brasil: ${ }^{1}$ Professor Titular, ${ }^{2}$ Estudante de Medicina, Bolsista de Iniciação Científica (PROBIC/FAPEMIG).

Recebido 22 Agosto 2001. Aceito 16 Outubro 2001.

Dr. José Eymard Homem Pittella - Laboratório de Neuropatologia, Departamento de Anatomia Patológica e Medicina Legal - Faculdade de Medicina, Universidade Federal de Minas Gerais - Av. Alfredo Balena 190 - 30130-100 Belo Horizonte MG - Brasil. E-mail: pittella@medicina.ufmg.br
} 
As doenças cerebrovasculares (DCVs) constituem a terceira causa de morte nos países desenvolvidos ${ }^{1}$, sendo responsáveis por $9-14,7 \%$ dos óbitos na população acima de 30-40 anos ${ }^{2,3}$. Além disso, observa-se aumento na incidência das DCVs com o avançar da idade, de 100/100000 indivíduos com idade entre 45-54 anos, para acima de 1800/100000 naqueles com idade superior a 85 anos $^{4}$. Como consequência, representam o grupo mais frequente de doenças do sistema nervoso central (SNC) no idoso $^{5-8}$. A mortalidade nos três primeiros meses após o acidente vascular cerebral (AVC) aumenta significativamente com a idade, variando de $11,1 \%$ entre 55 64 anos, $24 \%$ entre $65-74$ anos e $39,4 \%$ nos indivíduos com 85 ou mais anos ${ }^{9}$. Além disso, é comum a recorrência de AVCs, observada em $21 \%$ dos pacientes, a qual contribui para o aumento da morbidade pelo efeito cumulativo das lesões ${ }^{10}$. As DCVs são altamente incapacitantes para a população em geral e, em especial, para os idosos, que frequentemente são portadores de outras doenças cardiovasculares ${ }^{11}$ e possuem maior limitação para reabilitação após o AVC $^{9}$. As DCVs podem ainda produzir demência vascular, sendo esta a segunda causa de demência na maioria dos países (15-20\% dos casos), e a principal causa no Japão e na Rússia ${ }^{6,12}$, sua prevalência aumentando com a idade ${ }^{13}$.

Apesar do grande avanço tecnológico que vem ocorrendo nas últimas décadas nos métodos auxiliares de diagnóstico e, em particular, nos métodos de imagem, o diagnóstico estabelecido por tais métodos continua ainda sujeito a erros. O diagnóstico obtido à necropsia permanece o melhor método para determinar com precisão a natureza da doença de base e da causa da morte, especialmente nos casos de mortes inesperadas e súbitas ${ }^{14-16}$. Além disso, dados estatísticos de mortalidade devem ser interpretados com cautela quando o índice de confirmação pela necropsia é baixo, como é o caso em pacientes mais idosos ${ }^{3}$. Alguns estudos constataram discrepância de $8-16 \%$ entre o diagnóstico clínico e o neuropatológico dos $A V C^{5,17}$. Essa discrepância acentuase ainda mais quando são considerados os tipos de AVC e não apenas o diagnóstico genérico de AVC. Assim, hemorragia cerebral, infarto cerebral e hemorragia subaracnóidea foram diagnosticados corretamente em somente $55 \%, 51 \%$ e $38 \%$ dos casos, respectivamente ${ }^{17}$.

No Brasil, em decorrência da melhoria nas condições de vida e da crescente urbanização, especialmente a partir da segunda metade do século XX, tem ocorrido queda significativa nas taxas de mortalidade, aumento da expectativa de vida e envelhe- cimento da população ${ }^{18,19}$. Levando-se em conta esses aspectos e a inexistência de dados em nosso país, e os poucos existentes em outros países, sobre a frequência das DCVs em idosos com base em séries de necropsia ${ }^{3,5-8}$, resolveu-se conduzir o presente estudo com o objetivo de analisar a prevalência, os tipos e as repercussões clínicas das DCVs em indivíduos idosos, procedentes de um hospital geral, cujos diagnósticos foram estabelecidos através da necropsia, com avaliação neuropatológica detalhada.

\section{MÉTODO}

Foram analisados os laudos neuropatológicos arquivados no Laboratório de Neuropatologia do Departamento de Anatomia Patológica e Medicina Legal da Faculdade de Medicina da Universidade Federal de Minas Gerais (UFMG), em Belo Horizonte, Minas Gerais, dos pacientes com idade igual ou superior a 61 anos, procedentes em sua grande maioria do Hospital das Clínicas da UFMG e necropsiados consecutivamente no período 1976 a 1997. O Hospital das Clínicas é um hospital geral de ensino universitário e atende particularmente à população de baixa renda da região metropolitana de Belo Horizonte e de cidades próximas da zona metalúrgica do estado de Minas Gerais $^{20}$. Pequeno número de pacientes foi encaminhado de outros hospitais gerais de Belo Horizonte. Necropsias médico-legais não foram incluídas no estudo. Os prontuários clínicos dos pacientes selecionados foram revistos para avaliar a presença de sintomas e sinais neurológicos.

Os pacientes foram distribuídos segundo a década de vida e o sexo. Os diagnósticos neuropatológicos foram classificados conforme os grandes grupos de doenças do SNC (como cerebrovasculares, infecções, neoplasias). As DCVs foram classificadas de acordo com os subgrupos mais importantes (como aterosclerose, doença cerebrovascular hipertensiva, infarto, aneurisma) e distribuídas conforme o sexo e a década de vida dos pacientes, para avaliar se houve aumento da frequência com o avançar da idade. As DCVs mais frequentes, quando pertinente, foram analisadas também conforme a localização, número de lesões e estágio de evolução. A aterosclerose cerebral foi graduada conforme o grau de obstrução macroscópica das artérias que constituem o polígono de Willis, prevalecendo para efeito de classificação o maior grau de obstrução, ou seja, grau leve, com obstrução da ordem de 1/3 da luz; grau moderado, com obstrução de mais de 1/3 a 2/3; grau intenso, com obstrução de mais de $2 / 3$ da luz. O diagnóstico da doença cerebrovascular hipertensiva ${ }^{21}$ baseou-se no achado de uma ou mais lesões cerebrais (infarto lacunar; lesões vasculares no segmento distal das artérias lentículo-estriadas e lentículo-ópticas e nos ramos penetrantes das artérias pontinas; doença de Binswanger; hematoma cerebral) em pacientes portadores de hipertensão arterial e nos quais à necropsia havia nefrosclerose vascular benigna e/ou maligna e cardiopatia hipertensiva. As lesões vasculares cerebrais da hipertensão arterial foram 
Tabela 1. Distribuição por idade e sexo de 242 idosos procedentes de um hospital geral, necropsiados em Belo Horizonte, Minas Gerais, no período de 1976 a 1997.

\begin{tabular}{ccccccc}
\hline Idade & \multicolumn{2}{c}{ Feminino } & \multicolumn{2}{c}{ Masculino } & \multicolumn{2}{c}{ Total } \\
(em décadas) & $\mathrm{N}$ & $\%$ & $\mathrm{n}$ & $\%$ & $\mathrm{n}$ & $\%$ \\
\hline $61-70$ & 50 & 35,7 & 90 & 64,3 & 140 & 57,8 \\
$71-80$ & 30 & 41,1 & 43 & 58,9 & 73 & 30,2 \\
$81-90$ & 14 & 50,0 & 14 & 50,0 & 28 & 11,6 \\
$91-100$ & 1 & 100 & & & 1 & 0,4 \\
Total & 95 & 39,3 & 147 & 60,7 & 242 & 100 \\
\hline
\end{tabular}

classificadas segundo Rothemund \& Frische ${ }^{22}, Z$ ülch $^{23}$ e Ellison et al. ${ }^{4}$, tendo sido consideradas as duas mais características, quais sejam: fibroelastose intimal e hialinose (lipohialinose). Os infartos cerebrais foram classificados de acordo com o estágio evolutivo segundo Ellison et al. ${ }^{4}$. Em relação aos pacientes com encefalopatia hipóxicoisquêmica somente foram incluídos aqueles com necrose cortical cerebral difusa. Na presente série não foi incluída a angiopatia amilóide cerebral pelo fato de apenas em poucos pacientes ter sido realizada a coloração pelo Vermelho-Congo para identificar a presença da substância amilóide.

Os diagnósticos neuropatológicos foram previamente estabelecidos pelo mesmo neuropatologista (JEH Pittella) seguindo protocolo que incluía: 1) fixação do encéfalo em formol a $10 \%$ por aproximadamente duas semanas; 2 ) dissecção dos vasos da base; 3 ) cortes seriados frontais dos hemisférios cerebrais, horizontais do tronco encefálico e sagitais do cerebelo, a intervalos de $1 \mathrm{~cm}$; 4) identificação e descrição das lesões; 5) coleta de um fragmento dos lobos frontal, parietal, temporal e occipital, núcleos da base, hipotálamo, tálamo, mesencéfalo, ponte, bulbo e cerebelo; 6) inclusão em parafina e microtomia, obtendo-se geralmente um corte histológico para cada fragmento coletado; 7) coloração pela hematoxilina-eosina e, quando necessário, Weil-Weigert (mielina), Glees-Marsland (axônio) e elástica van Gieson. As artérias do sistema carotidiano e vertebral em seu trajeto extracraniano (ao nível do pescoço) não foram examinadas.

Para avaliar a associação entre: 1) infarto cerebral e aterosclerose; 2) doença cerebrovascular hipertensiva e aterosclerose; 4) doença cerebrovascular hipertensiva e gravidade da aterosclerose; 3 ) infarto lacunar e fibroelastose e/ou hialinose das pequenas artérias cerebrais, foram comparadas as frequências dessas variáveis e a significância estatística das diferenças encontradas entre as proporções foi avaliada pelo teste do qui quadrado, calculando-se o odds ratio (OR). Foram considerados significativos os valores de $\mathrm{p} \leq 0,05$ a um nível de confiança de $95 \%$.

\section{RESULTADOS}

No período de 1976 a 1997 foram necropsiados 242 idosos, os quais correspondem a 9,9\% da popu- lação submetida a necropsia nesse período (2447 indivíduos). A distribuição por década de vida e sexo dos 242 idosos pode ser vista na Tabela 1. A maioria dos pacientes era do sexo masculino e tinha idade entre 61 e 70 anos. O principal grupo de doenças do SNC foi representado pelas DCVs, acometendo $71,9 \%$ dessa população de idosos (Tabela 2). As DCVs

Tabela 2. Frequência dos principais grupos de doenças do sistema nervoso central em 242 idosos procedentes de um hospital geral, necropsiados em Belo Horizonte, Minas Gerais, no período de 1976 a 1997.

\begin{tabular}{lcc}
\hline Grupo de doenças & $\mathrm{N}$ & $\%$ \\
\hline Doenças cerebrovasculares & 174 & 71,9 \\
Infecções* & 30 & 12,4 \\
Neoplasias** & 17 & 7,1 \\
Traumatismos crânio-encefálicos*** & 9 & 3,7 \\
Doenças nutricionais**** & 6 & 2,5 \\
Doenças degenerativas**** & 4 & 1,7 \\
\hline * neurocisticercose (17), leptomeningite purulenta (5), criptococose (2), \\
toxoplasmose (2), outras (4). \\
** primitivas (6), metastáticas (11). \\
*** hemorragia subdural crônica (5), contusão antiga (4). \\
**** encefalopatia de Wernicke (6). \\
***** doença de Alzheimer (2), doença de Parkinson (2).
\end{tabular}

Tabela 3. Frequência das principais doenças cerebrovasculares em 242 idosos procedentes de um hospital geral, necropsiados em Belo Horizonte, Minas Gerais, no período de 1976 a 1997.

\begin{tabular}{lcc}
\hline Doença cerebrovascular & $\mathrm{n}$ & $\%$ \\
\hline Ateroclerose & 148 & 61,2 \\
Doença cerebrovascular hipertensiva & 62 & 25,6 \\
Infarto cerebral & 36 & 14,9 \\
Hemorragia intracraniana espontânea* & 7 & 2,9 \\
Aneurisma sacular do polígono de Willis & 7 & 2,9 \\
Encefalopatia hipóxico-isquêmica & 6 & 2,5 \\
Arterite** & 2 & 0,8 \\
\hline
\end{tabular}

* excluída hemorragia associada com hipertensão arterial e ruptura de aneurisma. Nesse grupo foram incluídos hematoma cerebral associado com leucemia (1), trombocitopenia (2) e metástase (1) e hemorragia subdural aguda de causa indeterminada (3).

** poliarterite nodosa (1) e arterite de células gigantes (1) 
Tabela 4. Distribuição em percentagem das principais doenças cerebrovasculares, por década de vida, em 242 idosos procedentes de um hospital geral, necropsiados em Belo Horizonte, Minas Gerais, no período de 1976 a 1997.

\begin{tabular}{lcccc}
\hline Tipo de doença cerebrovascular & \multicolumn{4}{c}{ Década de vida } \\
& $61-70$ & $71-80$ & $81-90$ & $91-100$ \\
\hline Aterosclerose & 57,9 & 60,3 & 78,6 & 100 \\
Doença cerebrovascular hipertensiva & 25,7 & 23,0 & $34,3^{*}$ & \\
Infarto cerebral & 17,9 & 6,8 & 7,1 & \\
\hline
\end{tabular}

*O aumento da frequência da doença cerebrovascular hipertensiva a partir da década de 80 envolveu tanto as lesões das pequenas artérias quanto os infartos lacunares.

Tabela 5. Frequência dos principais tipos de doença cerebrovascular hipertensiva em 62 idosos procedentes de um hospital geral, necropsiados em Belo Horizonte, Minas Gerais, no período de 1976 a 1997.

\begin{tabular}{lcc}
\hline & $\mathrm{n}$ & $\%$ \\
\hline Lesões vasculares cerebrais & 52 & 83,9 \\
Infarto lacunar & 39 & 62,9 \\
Doença de Binswanger & 4 & 6,5 \\
Hematoma cerebral & 2 & 3,2 \\
\hline
\end{tabular}

mais frequentes foram: aterosclerose $(61,2 \%)$, doença cerebrovascular hipertensiva $(25,6 \%)$ e infarto cerebral (14,9\%), como mostrado na Tabela 3. Observou-se aumento da frequência da aterosclerose e da doença cerebrovascular hipertensiva com o avançar da idade, esta última apenas a partir da nona década; por outro lado, o infarto cerebral foi mais comum entre os 61-70 anos (Tabela 4). O aumento da frequência da aterosclerose com o avançar da idade deveu-se ao aumento considerável da frequência da aterosclerose nas mulheres, especialmente a partir da oitava década.

Dos 147 idosos do sexo masculino, 65,3\% eram portadores de aterosclerose, contra 54,7\% dos 95 idosos do sexo feminino. Em relação à gravidade da aterosclerose, em $52,0 \%$ dos pacientes era de grau leve, em $23 \%$ era de grau moderado e em $25 \%$ era de grau intenso. Houve aumento da gravidade da aterosclerose com a idade, sendo que na faixa etária de 61-70 anos, 71-80 anos e 81-90 anos a frequência da aterosclerose de grau moderado/intenso foi, respectivamente, de $38,9 \%, 50,4 \%$ e $60 \%$. Quanto à localização, as principais artérias acometidas, em ordem decrescente, foram as artérias basilar $(51,4 \%)$, cerebral média $(41,2 \%)$, carótida interna $(33,8 \%)$, cerebral posterior $(24,3 \%)$ e vertebral $(22,3 \%)$.

As lesões das pequenas artérias cerebrais e os infartos lacunares foram os tipos de doença cerebrovascular hipertensiva mais comumente encontrados, respectivamente, $83,9 \%$ e $62,9 \%$ (tabela 5 ). A fibro- elastose associada com a hialinose foi observada em $38,5 \%$ dos 52 pacientes hipertensos com lesão das pequenas artérias cerebrais, enquanto a fibroelastose e a hialinose, isoladamente, foram identificadas em outros $53,8 \%$ e $7,7 \%$ dos pacientes, respectivamente. Os infartos lacunares eram solitários em $50 \%$ dos pacientes e nos outros $50 \%$ havia dois ou mais infartos. As sedes preferenciais foram os núcleos da base (94,9\%), especialmente o putâmen, seguida do tálamo (35,9\%), substância branca do lobo frontal $(30,8 \%)$ e base da ponte $(28,2 \%)$. Houve associação significativa entre a presença de infartos lacunares e fibroelastose e/ou hialinose das pequenas artérias cerebrais $[p=0,000 ; O R=27,42(10,73-72,3)]$.

Cerca da metade (49\%) dos infartos cerebrais eram antigos, $30 \%$ eram recentes e $21 \%$ eram subagudos. Os infartos cerebrais eram solitários em $60 \%$ dos pacientes, $34,4 \%$ tinham dois infartos e $5,6 \%$ tinham mais de dois. Os hemisférios cerebrais foram a sede preferencial dos infartos; os lobos occipital $(47,2 \%)$, frontal $(38,8 \%)$ e parietal $(33,3 \%)$, os núcleos da base (33,3\%), o lobo temporal $(19,4 \%)$ e o cerebelo $(16,7 \%)$ sendo as regiões mais atingidas, em ordem decrescente. Dos sete aneurismas saculares do polígono de Willis, 5 eram rotos e 2 eram íntegros; todos eram solitários, sendo 4 localizados na artéria cerebral média, 2 na junção da cerebral anterior com a comunicante anterior e 1 na carótida interna.

Não se observou associação significativa entre a presença de infarto cerebral e aterosclerose $(p=0,51)$. Houve associação significativa entre a presença de doença cerebrovascular hipertensiva e aterosclerose cerebral $[p=0,000 ; O R=3,95(2,03-7,73)]$ e entre doença cerebrovascular hipertensiva e aterosclerose de grau intenso $[p=0,000 ; O R=3,61(1,43-9,23)]$.

A análise dos prontuários clínicos e dos laudos neuropatológicos dos pacientes mostrou que quando se consideram apenas as DCV que resultaram em lesão cerebral e/ou das meninges (infarto, encefalopatia hipóxico-isquêmica, hematoma cerebral, he- 
morragia subdural ou subaracnóidea, doença de Binswanger, totalizando 75 pacientes), $32(42,7 \%)$ e $13(17,3 \%)$ casos de DCVs foram, respectivamente, clinicamente sintomáticos e os responsáveis diretos pelo óbito. Dos 36 pacientes com infarto cerebral, $12(33,3 \%)$ apresentavam sintomas e sinais neurológicos e $7(19,4 \%)$ faleceram em consequência da DCV. Somente $4(10,3 \%)$ dos 39 infartos lacunares eram clinicamente sintomáticos. Os 5 aneurismas saculares rotos produziram manifestações neurológicas, 3 dos quais causaram a morte do paciente. Seis dos 9 pacientes com hemorragia intracraniana espontânea de etiologia e topografia variadas (excluída ruptura de aneurisma) apresentaram manifestações clínicas, 4 dos quais faleceram em consequência da hemorragia.

\section{DISCUSSÃO}

Antes de se discutir os resultados do presente trabalho, devem ser comentadas resumidamente as limitações de um estudo retrospectivo como o ora realizado. Sua confiabilidade depende da existência de laudos neuropatológicos detalhados e fundamentados em exames padronizados do SNC. Embora os diagnósticos neuropatológicos tenham sido estabelecidos pelo mesmo neuropatologista seguindo protocolo padrão, algumas limitações devem ser assinaladas: 1) a não dissecção das artérias do pescoço para avaliar a presença de aterosclerose nesses vasos; 2) a possibilidade do número de infartos lacunares estar subestimado, pela fato de algumas dessas lesões serem menores do que os cortes de $1 \mathrm{~cm}$ utilizados nos recortes dos encéfalos, podendo, portanto, não terem sido visualizadas na superfície de corte ${ }^{8,24}$; 3) a possibilidade do número de pacientes com hialinose estar igualmente subestimado, em razão da utilização de apenas um corte histológico para cada fragmento coletado, quando se sabe que essa lesão tem distribuição segmentar, sendo comumente necessário 20-30 cortes consecutivos do mesmo fragmento para identificá-la ${ }^{23}$; 4) a não avaliação da angiopatia amilóide cerebral pelo fato de apenas em poucos pacientes ter sido realizada a coloração pelo Vermelho-Congo para identificar a presença da substância amilóide; 5) finalmente, a análise dos dados clínicos dos pacientes depende, igualmente, da atenção e persistência do médico em examinar o paciente e valorizar a presença de sintomas por vezes leves e passageiros, e da cooperação do paciente em relatá-los ${ }^{8,24}$.

São poucos os trabalhos existentes na literatura que estudam a frequência das DCVs em populações com idade superior a 60 anos $^{3,5-8}$ e são raros os que avaliam mais detalhadamente essas doenças nessa mesma população de idosos ${ }^{6,8}$, nesse último estudo tendo sido analisados somente os infartos cerebrais. O grupo das DCVs foi o mais frequente dos grupos de doenças do SNC na população de idosos analisada no presente trabalho $(71,9 \%)$, seguido pelas infecções do SNC (14,9\%). Os outros grupos de doença tiveram frequência baixa, principalmente o das doenças degenerativas $(1,7 \%)$, possivelmente pelo fato da amostra estudada ser proveniente de um hospital geral e não de instituições de longa permanência ou hospital geriátrico, onde a frequência da doença de Alzheimer e de outras doenças degenerativas é muito maior ${ }^{25}$. Apesar das necropsias médico-legais terem sido excluídas do presente estudo, traumatismos crânio-encefálicos foram encontrados em $3,7 \%$ dos idosos examinados, representados por hematoma subdural crônico e contusão antiga. O hematoma subdural crônico ocorre mais frequentemente em pacientes idosos com atrofia cerebral. 0 trauma pode ser inclusive de pequena intensidade, mas devido à relativa baixa pressão do espaço subdural, o sangue acumula com pouca compressão, fenômeno esse facilitado pela maior capacidade de acomodação do cérebro atrófico ${ }^{26}$, explicando assim sua presença em séries de necropsia de pacientes procedentes de um hospital geral. Contusão antiga foi observada em $2,5 \%$ de 2 mil necropsias de um hospital geral ${ }^{27}$, o que confirma o conceito de que as contusões são lesões geralmente compatíveis com boa evolução clínica, quando não associadas com outras lesões crânio-encefálicas traumáti$\mathrm{cas}^{28}$.

A aterosclerose cerebral foi a DCV mais frequente na presente série, acometendo cerca de $60 \%$ dos idosos examinados. Além disso, foi mais comum no sexo masculino, embora tenha havido aumento considerável da frequência da aterosclerose nas mulheres com o avançar da idade, e sua frequência e gravidade aumentaram igualmente com a idade. Em uma grande série de 1739 pacientes com mais de 61 anos de idade, Resch \& Baker ${ }^{29}$ não encontraram diferenças apreciáveis na frequência da aterosclerose cerebral entre os dois sexos. Entretanto, Resch et al. ${ }^{30}$ e Flora et al. ${ }^{31}$, numa série muito maior, observaram aumento da frequência e da gravidade da aterosclerose cerebral nas mulheres a partir da oitava década. A graduação macroscópica da aterosclerose cerebral, utilizada no presente trabalho, embora sujeita a alguma imprecisão ${ }^{32}$ e certamente menos exata do que o sistema proposto por outros auto- 
res $^{11,29}$, baseou-se em critérios definidos e semi-quantitativos e foi empregada pelo mesmo observador, tornando-a reproduzível. Resch \& Baker ${ }^{29}$, nos Estados Unidos, observaram que $86,1 \%$ dos indivíduos acima de 60 anos eram portadores de aterosclerose cerebral, havendo aumento da frequência e gravidade com a idade. Segundo esses autores, aterosclerose de grau intenso nas faixas etárias de 61-70 anos, 71-80 anos, 81-90 anos e acima de 90 anos foi observada em $40,4 \%, 60,9 \%, 76,3 \%$ e $91,3 \%$ dos casos, respectivamente. Diferenças quantitativas na frequência e gravidade da aterosclerose cerebral entre grupos étnicos distintos são conhecidas ${ }^{23,30,33-37}$, as quais em parte dependem do fato de que muitos dos fatores de risco da aterosclerose são determinados pelos hábitos de vida e condições sócio-econômicas ${ }^{1}$. A maior frequência e gravidade da aterosclerose cerebral nos norte-americanos em relação aos achados da presente série podem ser talvez explicadas por diferenças ambientais, particularmente por fatores dietéticos. A aterosclerose cerebral afeta mais intensamente as artérias carótidas internas, cerebrais médias, basilar, cerebrais posteriores e vertebrais ${ }^{23,29,38}$ e essas localizações foram as mais frequentemente encontradas na presente série. Apesar das artérias do sistema carotidiano e vertebral em seu trajeto extracraniano não terem sido examinadas, sabe-se que esses vasos são comumente atingidos pela aterosclerose ${ }^{38}$.

A doença cerebrovascular hipertensiva foi a segunda DCV mais frequente no presente estudo $(25,6 \%)$, achado esse que pode ser explicado pela elevada frequência da hipertensão arterial na população, atingindo, na maioria dos países, cerca de $20 \%$ da população adulta ${ }^{39}$. Além disso, apesar dos avanços no tratamento da hipertensão arterial, existe, ainda, grande dificuldade no controle adequado da hipertensão arterial em grandes faixas da população brasileira, seja porque a maioria dos casos permanece sem ser diagnosticada, seja pela não-aderência ou não-observância ao tratamento proposto, de ocorrência comum, especialmente nos pacientes assintomático ${ }^{39,40}$. Em consequência, há manutenção dos níveis pressóricos elevados e aparecimento e/ou agravamento das lesões vasculares cerebrais associadas com a hipertensão arterial. É difícil comparar os achados da presente série com os da literatura, pois não há estudo que avalie numa mesma população necropsiada os diferentes tipos de lesão vascular e cerebral associados com a hipertensão arterial. As lesões das pequenas artérias penetrantes cerebrais, fibroelastose intimal e/ou hialinose, foram as lesões mais comumente associadas com a hipertensão arterial, acometendo mais de $80 \%$ dos pacientes com doença cerebrovascular hipertensiva. Hialinose das pequenas artérias dos núcleos da base foi encontrada em $47 \%$ de 281 pacientes portadores de hipertensão arterial, sua frequência aumentando com a duração da hipertensão ${ }^{22}$. Se no presente estudo for considerado somente os pacientes com hialinose, essa lesão foi observada em $38,7 \%$ dos pacientes com doença cerebrovascular hipertensiva.

Na presente série, o infarto lacunar foi observado em $16,1 \%$ dos idosos e constituiu o segundo tipo de doença cerebrovascular hipertensiva mais comumente encontrado, acometendo $62,9 \%$ desses pacientes. Em duas séries de 1042 e 2859 necropsias consecutivas de adultos, com estudo neuropatológico detalhado, infartos lacunares foram identificados em $11 \%$ e $6 \%$ dos pacientes, respectivamente 24,41 , dos quais $72,1 \%$ tinham mais de 60 anos de idade $^{41}$. Os infartos lacunares ocorreram mais comumente na sétima e oitava décadas e sua maior frequência no presente estudo, comparada à relatada por Fisher ${ }^{41}$ e Tuszynski et al. ${ }^{24}$, pode ser explicada pelo fato de termos incluído somente indivíduos acima de 61 anos de idade. Em outra série de 966 indivíduos adultos com mais de 40 anos submetidos a necropsia foram observados $12,9 \%$ infartos cerebrais assintomáticos, sua frequência aumentando com a idade $^{8}$. Uma parcela significativa dessas lesões, pela localização, dimensões (menores do que $1 \mathrm{~cm}$ de diâmetro) e associação com níveis pressóricos mais elevados, poderia ser classificada como infartos lacunares. Diferentemente de Fisher ${ }^{41}$ que encontrou apenas $25,4 \%$ de infartos lacunares solitários, na presente investigação, os infartos lacunares eram solitários em $50 \%$ dos pacientes, frequência essa próxima aos $46 \%$ relatados por Tuszynski et al. ${ }^{24}$. A sede preferencial dos infartos lacunares no nosso estudo foi os núcleos da base (94,9\%), especialmente o putâmen, seguida do tálamo (35,9\%), substância branca do lobo frontal $(30,8 \%)$ e base da ponte $(28,2 \%)$, distribuição essa em linhas gerais similar à descrita por Fisher ${ }^{41}$ e Tuszynski et al. ${ }^{24}$. A associação significativa entre a presença de infartos lacunares e fibroelastose e/ou hialinose das pequenas artérias cerebrais, observada na presente série, havia sido previamente relatada por Rothemund \& Frische ${ }^{22}$ que encontraram infartos lacunares nos núcleos da base em $67 \%$ dos pacientes com hialinose. A hipertensão arterial é o principal fator de risco para o aparecimento de infartos lacunares, sendo encontrada em $64-97 \%$ dos pacientes com infartos lacunares ${ }^{24,41}$. 
Alguns autores relatam aumento da frequência da fibroelastose intimal com a idade, especialmente nos pacientes com hipertensão arterial ${ }^{42}$. Além disso, a incidência da hipertensão arterial aumenta com o envelhecimento ${ }^{43}$ e, conforme já mencionado, Shinkawa et al. ${ }^{8}$ notaram aumento da frequência de infartos lacunares com a idade, o maior número de casos ocorrendo após os 80 anos. Assim, é possível que o aumento da frequência da doença cerebrovascular hipertensiva a partir da nona década, envolvendo tanto as lesões das pequenas artérias quanto os infartos lacunares, na presente série, possa estar refletindo a maior incidência da hipertensão arterial nos idosos e o efeito deletério da manutenção, por longo período, de níveis pressóricos elevados sobre as pequenas artérias cerebrais ${ }^{39}$.

O hematoma cerebral associado com a hipertensão arterial foi pouco frequente na presente série, representando $0,8 \%$ das DCVs do idoso e $3,2 \%$ dos casos de doença cerebrovascular hipertensiva. Em uma série de 1721 necropsias consecutivas de indivíduos acima de 60 anos, hemorragia cerebral associada com hipertensão arterial foi identificada em 2,6\% dos idosos e em $8,2 \%$ daqueles com DCV clinicamente sintomática ${ }^{6}$. A hipertensão arterial é responsável por $40-50 \%$ dos casos de hemorragia cerebral não-traumática ${ }^{1,4}$. Essas lesões ocorrem particularmente entre os 40-69 anos de idade e localizam-se nos núcleos da base em $67-72 \%$ dos pacientes ${ }^{23,44}$. Diferentes estudos em diversos países, inclusive no Brasil, têm demonstrado redução na incidência e na mortalidade dos acidentes vasculares cerebrais isquêmicos e hemorrágicos, particularmente nos pacientes com hemorragia cerebral, a partir da década de $1950^{2,3,16,45-48}$. Admite-se que os avanços no tratamento e controle da hipertensão arterial, detecção de casos não fatais de hemorragia cerebral pela tomografia computadorizada e prevenção e tratamento mais adequados das complicações dos AVCs (p. ex., pneumonia de aspiração e encefalopatia hipóxico-isquêmica) sejam as principais causas para explicar esse achado ${ }^{2,45,46}$. A baixa frequência da hemorragia cerebral associada com hipertensão arterial, no presente estudo, frente ao elevado número de pacientes com doença cerebrovascular hipertensiva, pode ser explicada pelas características do Hospital das Clínicas da UFMG, de onde procedeu a grande maioria dos pacientes, qual seja, a de tratar-se de um hospital que não possuía um serviço de atendimento de urgência até no ano de 1996 e, como consequência, onde eram então pequenas as possibilidades de internação de pacientes com AVC.
A doença de Binswanger ocorreu em 1,7\% dos idosos necropsiados no presente estudo, correspondendo a $6,5 \%$ dos pacientes com doença cerebrovascular hipertensiva e a 5,3\% daqueles com DCV clinicamente sintomática. Em 1721 necropsias consecutivas de indivíduos acima de 60 anos, a doença de Binswanger foi observada em 3,5\% dos idosos necropsiados e em $11 \%$ dos pacientes com DCV sintomática ${ }^{6}$. A doença de Binswanger é um tipo de demência vascular, associada quase sempre com a hipertensão arterial e com maior frequência acima de 80 anos $^{6,13,49}$, explicando-se assim sua presença em séries de necropsias consecutivas de idosos.

Hemorragias intracranianas espontâneas (hematoma cerebral e hemorragia subdural), excluídas as causadas por hipertensão arterial e ruptura de aneurisma, foram encontradas em $2,9 \%$ dos idosos da presente série, representando $4 \%$ das DCVs. Essas lesões estavam associadas com leucemia, metástase, trombocitopenia e outros distúrbios da coagulação, que são causas conhecidas de hemorragia intracraniana não-traumática ${ }^{1,4,23,44}$, responsáveis por $18 \%$ dos casos ${ }^{1}$.

O infarto cerebral foi a terceira DCV mais comum no presente estudo $(13,2 \%$ dos idosos e $18,4 \%$ das DCVs), frequência essa muito inferior à relatada por Aronson \& Aronson $^{50}$ e Yamanouchi et al. ${ }^{6}$, 29\% e $23,8 \%$ dos idosos, respectivamente. Segundo esses últimos autores, os infartos cerebrais representaram $74,5 \%$ das DCVs. Yamanouchi et al. ${ }^{6}$ incluíram os infartos lacunares na categoria dos infartos e se na presente série for adotado o mesmo procedimento, a frequência de infartos cerebrais é de $29,3 \%$ dos idosos e $40,8 \%$ das DCVs. Em outras séries a incidência de infartos cerebrais clinicamente sintomáticos é de $26,2 \%$ dos adultos e idosos necropsiados ${ }^{8}$, constituindo a DCV sintomática mais comum. O infarto cerebral é mais comum entre os $60-90$ anos $^{6,8,23,44,50}$, os trabalhos mais recentes demonstrando aumento de sua frequência com a idade. Na série em estudo, $49 \%$ dos infartos cerebrais eram antigos, $34,7 \%$ eram recentes e $16,3 \%$ eram subagudos, indicando que pelo menos cerca da metade dos infartos em idosos são compatíveis com sobrevida. Os infartos cerebrais eram solitários em $56,2 \%$ dos pacientes, 37,5\% tinham dois infartos e $6,3 \%$ tinham mais de dois. Na série de Shinkawa et al. ${ }^{8}, 47 \%$ dos pacientes com infartos clinicamente sintomáticos possuiam três ou mais infartos cerebrais. Os infartos cerebrais ocorrem preferencialmente no território da artéria cerebral média ${ }^{23,44}$, os núcleos da base, os lobos frontal, parietal e temporal, o cerebelo e a pon- 
te sendo as regiões mais atingidas, em ordem decrescente $^{50}$. Na presente série, deve ser destacado que o lobo occipital foi o mais afetado e os núcleos da base ocuparam a terceira posição, junto com o lobo parietal. A proporção infarto/hemorragia cerebral, na presente série, de 3,6:1, é menor do que a de 10:1 relatada para os EUA e Alemanha ${ }^{4,23}$ e mais próxima daquela descrita para o Japão - cerca de $4: 1^{6}$.

Um último aspecto a ser ainda comentado em relação aos infartos cerebrais, incluindo os lacunares, é a possível relação da multiplicidade dessas lesões, encontrada com grande frequência na presente série e na literatura, com a recorrência dos acidentes vasculares cerebrais, especialmente os de natureza isquêmica, observada em $21 \%$ dos pacientes ${ }^{10}$. Apesar de não poder ser excluída a possibilidade do mesmo paciente ter tido mais de um infarto simultaneamente, a análise das causas, dos fatores de risco e da baixa mortalidade dos AVCs isquêmicos indica que uma parcela significativa dos infartos múltiplos pode ter ocorrido em momentos diferentes da vida do paciente.

Aneurismas saculares do polígono de Willis foram identificados em $2,9 \%$ dos idosos da presente série ( $4 \%$ das DCVs), dos quais 4 eram rotos e 3 íntegros, todos sendo solitários. A prevalência dos aneurismas saculares em estudos retrospectivos de necropsia é de $0,4 \%$, contra $3,6 \%$ da relatada em estudos prospectivos, e aumenta com a idade, a grande maioria sendo encontrada na faixa etária entre $60-79$ anos $^{51}$. Cerca de $75-80 \%$ dos aneurismas são solitários e as localizações encontradas na presente série correspondem àquelas mais comumente descritas ${ }^{43,44-51}$.

A associação significativa entre a presença da doença cerebrovascular hipertensiva e maior frequência e gravidade da aterosclerose cerebral, observada na presente série, havia sido relatada por Rothemund \& Frische ${ }^{22}$, que observaram maior frequência e gravidade da aterosclerose cerebral nos portadores de hialinose. Por outro lado, já era conhecida a associação entre hipertensão arterial e maior gravidade da aterosclerose cerebral ${ }^{38} \mathrm{e}$ a associação entre infarto lacunar e maior frequência e gravidade da aterosclerose cerebral ${ }^{41}$. Apesar de no presente estudo não ter sido evidenciada associação significativa entre a presença de infarto cerebral e aterosclerose, outros autores têm demonstrado que $64 \%$ dos pacientes com infarto cerebral de origem nãoembólica mostram aterosclerose cerebral com obstrução de $50 \%$ ou mais da luz ${ }^{6}$.
A frequência de DCVs clinicamente sintomáticas na presente série $(42,7 \%)$ foi superior à observada por Yamanouchi et al. ${ }^{6}$ em pacientes necropsiados em um hospital geriátrico no Japão (32\%). Do mesmo modo, a proporção de DCVs como causa de óbito $(17,3 \%)$ foi superior à relatada por vários autores: $6-13 \% \%^{5-7,24}$. Na presente série, $66,7 \%$ dos infartos cerebrais eram assintomáticos, dados esses similares aos registrados por Aronson \& Aronson $^{50}$ que, numa grande série de 4003 adultos e idosos necropsiados, encontraram infartos cerebrais em cerca de $25 \%$ dos casos, a maioria dos quais representava achado de necropsia. Quando clinicamente sintomáticos os infartos cerebrais causam frequentemente a morte do paciente: assim, sete dos 12 pacientes da presente série e $52,6 \%$ dos 258 pacientes com infarto cerebral analisados por Shinkawa et al. ${ }^{8}$ faleceram em decorrência da DCV. Por outro lado, Yamanouchi et al. ${ }^{6}$ relataram que apenas $6,6 \%$ dos 410 infartos cerebrais clinicamente sintomáticos em idosos foram os responsáveis diretos pela morte do paciente. No que diz respeito a baixa frequência $(10,3 \%)$ de manifestações neurológicas nos infartos lacunares, os achados da presente série estão em linhas gerais de acordo com os da literatura: somente $22,8 \%$ de 114 pacientes ${ }^{41}$ e $19 \%$ de 169 pacientes ${ }^{24}$ com infartos lacunares apresentavam sintomas e sinais neurológicos.

\section{REFERÊNCIAS}

1. Kalimo H, Kaste M, Haltia M. Vascular diseases. In Graham DI, Lantos PL (eds). Greenfield $\$$ s Neuropathology. 6.Ed.Vol I. London: Arnold, 1997:315-396.

2. Torvik A, Stenwig JT. Changes in frequency of cerebrovascular diseases in Oslo, Norway, 1958-1977. Stroke 1981;12:816-823.

3. Karwinski B. Cerebrovascular disease at autopsy and in the death statistics for the county of Hordaland, Norway. APMIS 1993;101:269-274.

4. Ellison D, Love S, Chimelli L, et al. Neuropathology: a reference text of CNS pathology. London: Mosby, 1998:9.1-9,28;10.1-10.25.

5. Gross JS, Neufeld RR, Libow LS, Gerber I, Rodstein M. Autopsy study of the elderly institutionalized patient; review of 234 autopsies. Arch Intern Med 1988;148:173-176.

6. Yamanouchi H, Shimada H, Kuramoto K. Subtypes and proportions of cerebrovascular diseases in na autopsy series in a Japanese geriatric hospital. Klin Wochenschr 1990;68:1173-1177.

7. Mac Gee W. Causes of death in a hospitalized geriatric population: an autopsy study of 3000 patients. Virchows Arch A 1993;423:343-349.

8. Shinkawa A, Ueda K, Kiyohara Y, et al. Silent cerebral infarction in a community-based autopsy series in Japan. Stroke 1995;26: 380-385.

9. Nakayama H, Jorgensen HS, Raaschou HO, Olsen TS. The influence of age on stroke outcome. The Copenhagen study. Stroke 1994;25:808-813.

10. Sacco RL, Wolf PA, Kannel WB, McNamara PM. Survival and recurrence following stroke. The Framingham study. Stroke 1982;13:290-295.

11. Baker AB, Iannone A, Kinnard J. Cerebrovascular disease: VI. Relationship to disease of the heart and the aorta. Neurology 1961;11:63-70.

12. Jorm AF, Korten AE, Henderson AS. The prevalence of dementia: a quantitative integration of the literature. Acta Psychiatr Scand 1987; 76:465-479.

13. Esiri MM, Hyman BT, Beyreuther K, Masters CL. Ageing and dementia In Graham DI, Lantos PL (eds). Greenfieldథs Neuropathology. 6.Ed. Vol II. London: Arnold, 1997:153-233.

14. Kircher T, Nelson J, Burdo H. The autopsy as a measure of accuracy of the death certificate. N Engl J Med 1985;313:1263-1269. 
15. McKelvie PA. Medical certification of causes of death in an Australian metropolitan hospital: comparison with autopsy findings and a critical review. Med J Aust 1993;158:816-821.

16. Lahti RA, Sarna S, Penttilä A. Exploitation of autopsy in determining natural cause of death: trends in Finland with special reference to the diagnostics of ischemic heart diseases and cerebrovascular diseases in middle-aged males, 1974-1993. Forensic Sci Int 1998;91:109-121.

17. Hasuo Y, Ueda K, Kiyohara Y, et al. Accuracy of diagnosis on death certificates for underlying causes of death in a long-term autopsy-based population study in Hisayama, Japan: with special reference to cardiovascular diseases. J Clin Epidemiol 1989;42:577-584.

18. Cançado F. Epidemiologia do envelhecimento. In Cançado F (Ed.). Noções práticas de geriatria. Belo Horizonte: Coopmed Editora/Health CR, 1994:15-43.

19. Chaimowicz F. Os idosos brasileiros no século XXI: demografia, saúde e sociedade. Belo Horizonte: Postgraduate, 1998:23-41.

20. Vieira MI, Petroianu. A procedência de pacientes tratados em hospitais de "referência" em Belo Horizonte. Rev Med Minas Gerais 1995; 5:12-14.

21. Weller RO. Spontaneous intracranial haemorrhage. In Hume Adams J, Duchen LW (Eds). Greenfield $\Phi_{s}$ Neuropathology. 5.Ed. New York: Oxford Univ Press, 1992:269-301.

22. Rothemund E, Frische M. Klinisch-pathologische Studie zur Entstehung der intracerebralen Gefässhyalinose bei Hipertonie. Arch Psychiat Nervenkr 1973;217:195-206.

23. Zülch KJ. Cerebrovascular pathology and pathogenesis as a basis of neuroradiological diagnosis. In Diethelm L, Heuck F, Olsson O, Strnad F, Vieten H, Zuppinger A (Eds). Handbuch der medizinischen Radiologie. Band XIV, Teil 1A. Berlin: Springer Verlag, 1981:1-192.

24. Tuszynski MH, Petito CK, Levy DE. Risk factors and clinical manifestations of pathologically verified lacunar infarctions. Stroke 1989;20:990-999.

25. Ince PG, McArthur FK, Bjertness E, Torvik A, Candy JM, Edwardson JA. Neuropathological diagnoses in elderly patients in Oslo: Alzheimer\$s disease, Lewy body disease, vascular lesions. Dementia 1995; 6:162-168.

26. Brocklehurst G. Diagnoses not to be missed: subdural haematoma. Br J Hosp Med 1982;27:170-174.

27. Welte E. Über die Zusammenhänge zwischen anatomischem Befund und klinischem Bild bei Rindenprellungsherden nach stumpfen Schädeltrauma. Arch Psychiat Nervenkr 1948;179:243-315.

28. Pittella JEH, Gusmão SNS. Patologia do trauma cranioencefálico. Rio de Janeiro: Revinter, 1995:21-31.

29. Resch JA, Baker AB. Etiologic mechanisms in cerebral atherosclerosis: preliminary study of 3,839 cases. Arch Neurol 1964;10:617-628.

30. Resch JA, Okabe N, Loewenson R, Kimoto K, Katsuki S, Baker AB. A comparative study of cerebral atherosclerosis in a Japanese and Minnesota population. J Atheroscler Res 1967;7:687-693.

31. Flora GC, Baker AB, Loewenson RB, Klassen AC. A comparative study of cerebral atherosclerosis in males and females. Circulation 1968; 38:859-869.

32. Giertsen JC. The reliability of macroscopical grading of atherosclerosis. Acta Path Microbiol Scand 1960;50:355-368.
33. Baptista AG. Studies on the arteries of the brain: I. Etiologic factors in cerebral atherosclerosis. Angiology 1962;13:367-371.

34. Baker AB, Flora GC, Resch JA, Loewenson R. The geographic pathology of atherosclerosis: a review of the literature with some personal observations on cerebral atherosclerosis. J Chron Dis 1967;20:685-706.

35. Solberg LA, McGarry PA, Moossy J, et al. Distribution of cerebral atherosclerosis by geographic location, race, and sex. Lab Invest 1968; 18:604-612.

36. Williams AO, Resch JA, Loewenson RB. Cerebral atherosclerosis: a comparative autopsy study between Nigerian Negroes and American Negroes and Caucasians. Neurology 1969;19:205-210.

37. Gorelick PB. Distribution of atherosclerotic cerebrovascular lesions: effects of age, race, and sex. Stroke 1993;24(Suppl 1):16-19.

38. Fisher CM, Gore I, Okabe N, White PD. Atherosclerosis of the carotid and vertebral arteries: extracranial and intracranial. J Neuropath Exper Neurol 1965;24:455-476.

39. Williams GH. Hypertensive vascular disease. In Fauci AS, Braunwald E, Isselbacher KJ, et al. (Eds). Harrison's Principles of Internal Medicine. 14.Ed. Tradução em português. Rio de Janeiro: McGraw-Hill, 1998:14721486.

40. Rocha MOC, Teixeira R. Hipertensão arterial. In Pedroso ERP, Rocha MOC, Silva OA (Eds). Clínica médica: os princípios da prática ambulatorial. São Paulo: Atheneu, 1993:723-743.

41. Fisher CM. Lacunes: small, deep cerebral infarcts. Neurology 1965; 15:774-784.

42. Brierley JB, Graham DI. Hypoxia and vascular disorders of the central nervous system. In Hume Adams J, Corsellis JAN, Duchen LW (Eds). Greenfieldథs Neuropathology. 4.Ed. London: Edward Arnold, 1984:125207.

43. Locksley HB. Report of the cooperative study of intracranial aneurysms and subarachnoid hemorrhage: Section V, Part I. Natural history of subarachnoid hemorrhage, intracranial aneurysms and arteriovenous malformations. Based on 6368 cases in the cooperative study. J Neurosurg 1966;25:219-239.

44. Stehbens WE. Pathology of the cerebral blood vessels. Saint Louis: Mosby, 1972:284-350; 351-470.

45. Whisnant JP. The decline of stroke. Stroke 1984;15:160-168.

46. Broderick JP, Phillips SJ, Whisnant JP, OÆFallon WM, Bergstralh EJ Incidence rates of stroke in the eighties: the end of the decline in stroke? Stroke 1989;20:577-582.

47. Bonita R, Stewart A, Beaglehole R. International trends in stroke mortality: 1970-1985. Stroke 1990;21:989-992.

48. Lotufo PA, Lolio CA. Tendência da mortalidade por doença cerebrovascular no Estado de São Paulo: 1970 a 1989. Arq Neuropsiquiatr 1993;51:441-446.

49. van Swieten JC, van den Hout JHW, van Ketel BA, Hijdra A, Wokke JHJ, van Gijn J. Periventricular lesions in the white matter on magnetic resonance imaging in the elderly: a morphometric correlation with arteriolosclerosis and dilated perivascular spaces. Brain 1991;114:761-774.

50. Aronson SM, Aronson BE. Ischemic cerebral vascular disease: frequency in autopsy population. N Y State J Med 1966;66:954-957.

51. Rinkel GJE, Djibuti M, Algra A, van Gijn J. Prevalence and risk of rupture of intracranial aneurysms: a systematic review. Stroke 1998;29:251-256. 